\section{DINÁMICA Y ESTRUCTURA DE LAS MIGRACIONES RESIDENCIALES INTERURBANAS EN CHILE, 1987-2002'}

Severino Escolano Utrilla² ${ }^{2}$ Jorge Ortiz Véliz ${ }^{3}$ y Rodrigo Moreno Mora ${ }^{4}$

\section{Resumen}

Durante el quinquenio 1997-2002, las migraciones residenciales entre las ciudades chilenas mayores de 50.000 habitantes mantuvieron la intensidad que tenían diez años antes frente al descenso de este tipo de movilidad que, en el mismo período, se registró entre las ciudades de tamaño inferior a este umbral. Mediante el modelado y la aplicación de métodos de análisis de redes a los flujos migratorios bilaterales entre ciudades -obtenidos a partir de microdatos censales-, se ha constatado que los movimientos residenciales interurbanos presentan una estructura muy polarizada y estable, pero compatible con una notable dinámica de

\section{DYNAMICS AND STRUCTURE OF INTERURBAN RESIDENTIAL MIGRATIONS IN CHILE, 1987-2002'}

Severino Escolano Utrilla ${ }^{2}$, Jorge Ortiz Véliz ${ }^{3}$ and Rodrigo Moreno Mora ${ }^{4}$

\section{Abstract}

Over the 1997-2002 period, residential migrations among Chilean cities with more than 50,000 inhabitants maintained the intensity they had had ten years earlier which contrasts with the decrease in this type of mobility in smaller cities during the same period under review. Through the modeling and implementation of network analysis methods to the bilateral migratory flows between cities -collected through census microdata- it is revealed that interurban residential mobility is highly polarized, stable and compatible with a remarkable dynamic of changes in the direction and magnitude of flows. The spatial organization of interurban migrations 
cambios en la dirección y magnitud de los flujos. La organización espacial de las migraciones interurbanas comprende varios niveles y escalas, cuya jerarquía y articulación se asocian fuertemente a la configuración de la red urbana, es decir, a la distribución espacial de las ciudades, de la población y de la especialización de las actividades económicas. En el período y materia investigados, ha decrecido ligeramente la concentración de los destinos de la movilidad residencial, pero no se observa un proceso de descentralización intenso y generalizado.

\section{PALABRAS CLAVE: MIGRACIONES INTERURBANAS; MOVILIDAD RESIDENCIAL; RED URBANA; ANÁLISIS DE REDES; CHILE.}

Recibido: 27-02-2018

Aceptado: 01-10-2018

1 Este artículo se ha realizado con apoyo de un proyecto financiado por Ministerio de Economía, Industria y Competitividad, referencia CS020167488C43R.

2 España. Universidad de Zaragoza. ORCID: http://orcid.org/00000002-3489-0692. Correo electrónico: severino@unizar.es.

3 Chile. Profesor titular Departamento de Geografía, Facultad de Arquitectura y Urbanismo, Universidad de Chile. ORCID: http:// orcid.org/0000-0002-7951-6055. Correo electrónico: jortiz@ uchilefau.cl.

4 Chile. Profesor asistente Departamento de Geografía, Facultad de Arquitectura y Urbanismo, Universidad de Chile. ORCID: http://orcid.org/0000-0002-5351-2531. Correo electrónico: romoreno@uchilefau.cl. has different levels and scales, whose hierarchy and ways of integration are strongly associated with the configuration of urban networks, that is, the spatial distribution of cities, population and specialization of economic activities. Although the material from the period under review shows a slight decrease in the destinations for residential mobility, there are no signs of an intense and widespread decentralization process.

\section{KEYWORDS: INTERURBAN MIGRATIONS; RESIDENTIAL MOBILITY; URBAN NETWORK; NETWORK ANALYSIS; CHILE}

Received: 27-02-2018

Accepted: 01-10-2018

1 This research was funded by the Ministry of Economy, Industry and Competitiveness, Ref. CS02016-7488-C4-3-R.

2 Spain. University of Zaragoza. ORCID: http://orcid.org/00000002-3489-0692. Email: severino@unizar.es.

3 Chile. Professor, Department of Geography, Faculty of Architecture and Urban Planning, University of Chile. ORCID: http://orcid. org/0000-0002-7951-6055. Email: jortiz@uchilefau.cl.

4 Chile. Assistant Professor, Department of Geography, Faculty of Architecture and Urban Planning, University of Chile. ORCID: http://orcid.org/0000-0002-5351-2531. Email: romoreno@uchilefau.cl. 


\section{Introducción}

Los movimientos migratorios de la población forman parte importante de los principales procesos socioterritoriales de las sociedades contemporáneas a todas las escalas. Este trabajo se enmarca en el contexto de las migraciones internas, las que se producen en el interior de los espacios nacionales. Específicamente, trata de la dinámica y estructura recientes de la red de las migraciones residenciales interurbanas en Chile, que está configurada por los contingentes de personas que han cambiado de ciudad de residencia -en los períodos 1987-1992 y 1997-2002- y por las conexiones bilaterales entre las ciudades de origen y destino de los migrantes.

Desde el último tercio del siglo XX, las migraciones, especialmente las internacionales, han adquirido modalidades específicas de la actual etapa histórica, que se manifiestan, entre otros aspectos, en el considerable volumen de sus flujos, en las variadas causas y efectos que producen en los lugares de origen y destino, en su alcance global y en las múltiples modalidades que actualmente reviste el hecho migratorio (Artal-Tur, Peri y Requena, 2014; Castles, Haas y Miller, 2014; Imilan, Garcés y Margarit, 2014).

Las "nuevas complejidades" de las migraciones contemporáneas (Massey et al., 1998, p. 15) han impulsado la creación de marcos teóricos y metodológicos alternativos a la teoría neoclásica de las migraciones, insuficiente para explicar las migraciones actuales. A la concepción de las migraciones como un medio de ajuste de los desequilibrios espaciales de los mercados de trabajo o como una opción para mejorar las condiciones de vida de los migrantes, se han sumado nuevos argumentos para explicar las migraciones. Junto a los tradicionales factores de expulsión y atracción (push-pull), las nuevas teorías consideran también, entre otras dimensiones, las circunstancias personales, sociales y geográficas de los migrantes, las políticas migratorias de sus áreas de procedencia y destino o la existencia de redes personales y familiares de quienes emigran, además de las causas de los múltiples efectos que las migraciones producen en los lugares de origen y destino (Arango, 2000; Massey, Alarcón, Durand y González, 1990; Mora y Taylor, 2006). Entre los motivos para migrar, más o menos estructurales o coyunturales, figuran las condiciones ambientales, ocurrencia de desastres y de conflictos bélicos, étnicos o religiosos que fuerzan a desplazarse a millones de personas en todo el mundo.

Las migraciones interurbanas no han sido objeto de reflexión teórica ni de análisis empíricos tan amplios como las migraciones a otras escalas, aunque aquellas alimentan hoy flujos más cuantiosos que los de otras corrientes migratorias, especialmente en países como Chile, que tienen una elevada proporción de población urbana (86,5\% en 2002). 
El predominio de los movimientos migratorios ciudad-ciudad e intraurbanos, ya fue avanzado en la "hipótesis sobre la transición de la movilidad" (Zelinsky, 1971) para países que han finalizado su transición demográfica y han alcanzado un grado de modernización socioeconómica elevado.

Este tipo de migraciones presenta características propias, puesto que las ciudades, como centros de origen y destino de los flujos migratorios, se diferencian de otros espacios por el gran tamaño y diversidad de sus mercados laborales, por las mayores oportunidades para mejorar las condiciones de vida, reales o percibidas, por sus políticas sociales y de gobernanza, etc.; en suma: por los efectos producidos por la aglomeración, la gran diversidad y la densa conectividad de las ciudades (Taylor, 2017). A pesar de estas diferencias, la escasa investigación sobre las migraciones residenciales interurbanas se ha llevado a cabo a partir de los marcos teóricos y metodológicos generales para el estudio de las migraciones, no obstante, algunos autores han subrayado la influencia de la estructura de las redes urbanas en este tipo de migraciones (Cadwallader, 1992, p. 47; Pumain y Saint-Julien, 1990).

Por otro lado, las investigaciones recientes han destacado la gran importancia explicativa que las redes interpersonales de los migrantes tienen en las causas, modalidades, continuidad y perpetuación de las migraciones (Massey et al., 1990). Las redes migratorias figuran entre los principales factores explicativos de las migraciones actuales y, por tanto, ocupan una posición central de la investigación actual de las migraciones (Arango, 2000, p. 291 y ss). Además, las redes migratorias entroncan con la teoría del capital social, pues la configuración de las redes interpersonales limita o facilita las condiciones de acceso de las personas a mercados de bienes y servicios (Lin, Cook y Burt, 2001; Putnam, Leonardi y Nanetti, 1994).

Aquí se acepta como hipótesis de trabajo que los factores explicativos indicados se pueden aprehender indirectamente a partir de la consideración de las ciudades del sistema urbano chileno como una red única, que los migrantes interpretan y practican mediante sus desplazamientos. La estructura de esta red de flujos migratorios se puede describir por dos tipos de principios: uno espacial, definido por la proximidad geográfica y otro topológico, generado por las conexiones entre la ciudad de origen y la de residencia actual de los migrantes. Ambos, proximidad y conectividad, sintetizan las decisiones de quienes emigran e, indirectamente, la combinación de factores económicos, sociales, geográficos, afectivos, familiares y otras circunstancias, como la tradición migratoria, que movilizan a los migrantes.

La conceptualización de las migraciones interurbanas como una red de conexiones implica que la dinámica y estructura de los flujos migratorios se desarrollan en el seno de una totalidad organizada, 
cuyos componentes -flujos migratorios, ciudadesestán ligados por sus interacciones. De aquí se deriva que las ciudades no son islas o pares reflejos, una de otra, en un esquema de relaciones bilaterales exclusivas. En consecuencia, el análisis de las propiedades de los modelos de redes de las migraciones interurbanas es muy útil para descubrir el comportamiento conjunto del sistema migratorio.

Con este contexto, el presente trabajo pretende responder a las siguientes preguntas: ¿Cuáles son las características fundamentales de la organización de la red de migrantes interurbanos? ¿Cuáles han sido los cambios más relevantes habidos en los períodos estudiados? ¿Cuáles son los patrones geográficos de la red migratoria?

Para dar respuesta a estas cuestiones se han seleccionado algunas propiedades de las redes, congruentes con el comportamiento del fenómeno migratorio, es decir, que retengan el carácter bilateral de los flujos, pero contemplados en la totalidad de la red. A partir de los resultados obtenidos se examinarán las siguientes proposiciones:

a) La red migratoria interurbana funciona como un conjunto único de ciudades, en el que se identifican estructuras generales y locales interconectadas. Esta organización es fruto de la actuación consistente y combinada de fuerzas de diferente alcance espacial. b) La organización de la red presenta asimetrías y diferencias, que se pueden caracterizar por su grado de centralización y dispersión, diversidad de orígenes y destinos, distancia geográfica recorrida y agrupaciones espaciales (clúster). Los valores de los indicadores revelan las tendencias hacia la descentralización o concentración del fenómeno migratorio interurbano.

Los resultados obtenidos contribuyen a ampliar el conocimiento de las migraciones interurbanas. En primer lugar, se aborda un tema poco estudiado, del que apenas existen trabajos empíricos. Además, se aplica un enfoque novedoso, el análisis de redes con información espacial, que permite considerar las migraciones interurbanas simultáneamente en el contexto geográfico -influencia de la distancia y proximidad, autocorrelación espacialy en el contexto social -similitudes en función del grado de vinculación entre los elementos (Luo y MacEachren, 2014, p. 29).

Las restantes secciones de este artículo se dedican al desarrollo de los siguientes contenidos. En primer lugar, se presentan algunos aspectos significativos para contextualizar las migraciones interurbanas en Chile. Después, se describen los datos y los métodos utilizados. A continuación, se exponen y valoran los resultados de los análisis. Finalmente, en las conclusiones se evalúan los resultados en relación con los objetivos e hipótesis planteados y se proponen futuras líneas de investigación. 


\section{El contexto de las migraciones interurbanas en Chile}

La dinámica de las migraciones interurbanas durante los períodos estudiados se relaciona con transformaciones importantes habidas en la sociedad y economía chilenas. Muy probablemente, la fuerte integración de la economía chilena en el sistema económico mundial, iniciada a mediados de la década de 1970 en el marco de un modelo socioeconómico neoliberal, constituye la principal fuerza impulsora de procesos de reestructuración social, económica y territorial del país, de gran alcance a todas las escalas.

Uno de los medios puestos en práctica para acelerar el desarrollo económico ha consistido en el incremento de la producción orientada a la exportación de productos de minería, la agricultura, de la explotación forestal y pesquera y de los servicios, principalmente (Henríquez y Venegas, 2007). Las grandes inversiones de capital extranjero y nacional, que se han diversificado por múltiples ámbitos productivos (Barton, Gwynne y Murray, 2007), se han concentrado en las regiones de Santiago, Antofagasta y Atacama, lo que ha fortalecido la base económica de algunas ciudades medias como Iquique, Antofagasta o Puerto Montt y la consiguiente inserción especializada de las ciudades y regiones en el sistema económico mundial. El mismo proceso ha beneficiado también a la capital, Santiago, que se ha convertido en la puerta de entrada al país, lo que ha reforzado su tradicional primacía (Di Filippo y Bravo, 1977).

Otro factor que influye en la movilidad de la población, vinculado también al crecimiento económico, ha sido la ampliación y la mejora continuada de las infraestructuras y servicios de transporte terrestre y aéreo (aeropuertos y aeródromos), así como de las comunicaciones, como medios indispensables para alcanzar algunas prioridades estratégicas de la política de desarrollo, como la de "mejorar la conectividad interna y externa" (Gobierno de Chile, 2001, p. 4). Así, se han realizado considerables inversiones para la reforma o creación de nuevos terminales aeroportuarios de la "red troncal nacional" (Iquique, Puerto Montt, La Serena, Calama, Concepción, Punta Arenas, Aeropuerto Internacional de Santiago). Además, entre 1990-1999 se construyeron $78.500 \mathrm{~m}^{2}$ de nuevas terminales y se han previsto aeropuertos adicionales (La Serena, Temuco, Copiapó (Gobierno de Chile, 2001, p. 25 y ss.). En el mismo sentido, también se ha mejorado de la red viaria: en la década de los noventa se transformaron en autopista $1.500 \mathrm{~km}$ de la Ruta 5 entre La Serena y Puerto Montt (Panamericana, eje vertebral del país). También se han arreglado las conexiones con puertos y se han llevado a cabo otras obras de reforma en la Ruta Costera y la Ruta Austral (Gobierno de Chile, 2001, p. 27 y ss.). Al 
mismo tiempo, ha crecido de forma continuada el parque de vehículos, en especial los vehículos privados: los hogares que disponen de vehículo privado crecieron el 88,6\%, entre 1992 y 2002 (Instituto Nacional de Estadísticas [INE], 2009b, p. 11). La mayor disponibilidad de infraestructuras y medios de transporte y comunicaciones amplía las opciones de movilidad, de tal forma que los desplazamientos pendulares a larga distancia pueden ser una alternativa a la migración residencial, más permanente.

Como en otros países latinoamericanos, una de las manifestaciones más visibles e importantes de los cambios socioeconómicos, se encuentra en la terciarización de la economía y sociedad. Los servicios constituyen una de las bases del desarrollo económico, en particular los relacionados con el sector financiero y con la creación, tratamiento y distribución de la información (por ejemplo, la principal aportación al PIB en 2007 procede de "los servicios financieros y empresariales", cuya participación alcanzó el 16,2\% del PIB, por delante de la industria manufacturera, del comercio y de la minería) (INE, 2009a, p. 276). Las actividades de servicios se concentran en Santiago y en las capitales regionales, y los "servicios intensivos en conocimiento" se localizan, preferentemente, en las grandes ciudades (Escolano y Ortiz, 2017). En general, los servicios, incluido el turismo, son actividades muy dinámicas e impulsoras de todo tipo de movimientos migratorios.
Finalmente, hay que subrayar que los procesos de cambio también han afectado a la organización territorial de la sociedad y la economía, una de cuyas transformaciones más transcendentes ha sido la formación de áreas metropolitanas (Hidalgo, Mattos y Arenas, 2009), que se han consolidado como centros de articulación de la red urbana nacional y de los espacios regionales (Escolano, 2012). Los nuevos tejidos urbanos se caracterizan por su dispersión espacial y su fragmentación física, social y funcional, cualidades que, a priori, tienden a incrementar los movimientos espaciales.

En resumen, la liberalización económica y el sólido anclaje de la sociedad y economía chilenas con el sistema mundial han producido fuertes impactos sociales económicos y territoriales en Chile. En conjunto, los procesos indicados han modificado las condiciones sociales, económicas, tecnológicas y espaciales de la movilidad, en el sentido de intensificar los movimientos interurbanos e intraurbanos, estos últimos con recorridos más largos.

\section{Datos y métodos}

Los flujos migratorios representan a las personas que han cambiado de ciudad de residencia durante los cinco años previos a la fecha de realización del Censo Nacional de Población y Vivienda 1992 y de 2002. Los datos más recientes, del censo de 2017, 
se publicaron en junio de 2018. La información se obtuvo de las respuestas dadas a la pregunta de ambos censos, para todas las personas de cinco años o más: "¿En qué comuna o lugar vivía usted en abril de 1997 (o 1987)?". La consulta infravalora las migraciones residenciales, pues excluye los movimientos habidos dentro del quinquenio e ignora los producidos en los primeros cinco años del período intercensal. No obstante, estos datos permiten las comparaciones en el tiempo así como de los resultados entre los diversos estudios en los que se utilizan como fuente.

Las ciudades se han definido a partir del conjunto de zonas (entidad espacial del Censo Nacional de Población y Viviendas) de un municipio, calificadas como urbanas en el censo, contiguas o no, que suman más de 50.000 habitantes en 2002. La muestra se compone de 44 ciudades en 1992, que suponen el $58,8 \%$ de la población total del país y de 46 ciudades en 2002, que significan el 62,1\% de la población total.

La organización de los datos y su proceso analítico se pueden agrupar en tres bloques. El primero ha consistido en la elaboración de una matriz para cada período: en las filas, se recogen los contingentes de personas que salen de una ciudad para fijar su residencia en otras ciudades; en las columnas, se reflejan las personas que han fijado su residencia en una ciudad, procedentes de las demás ciudades. A partir de esta información básica se han derivado los siguientes cocientes: a) proporción de emigrantes de una ciudad que residen en otra ciudad $\left.\left(\mathrm{s}_{i j} / \sum_{j}^{\mathrm{n}} \mathrm{s}\right) ; \mathrm{b}\right)$ proporción de migrantes de una ciudad en relación con el total de migrantes de la ciudad de destino $\left.\left(\mathrm{s}_{\mathrm{ij}} / \sum_{\mathrm{i}}^{\mathrm{m}} \mathrm{s}\right) ; \mathrm{c}\right)$ proporción de migrantes de una ciudad en relación con el total población de la ciudad $\left(\mathrm{s}_{i j} / \sum_{j}^{\mathrm{n}} \mathrm{s} /\right.$ población total de una ciudad en un año determinado) y d) proporción de personas inmigrantes de una ciudad en relación con la población de la ciudad ( $\sum_{\mathrm{i}}^{\mathrm{m}} \mathrm{s} /$ población total de la ciudad en un año determinado). La comparación de los totales de fila con los totales de columna correspondientes a la misma ciudad, proporciona el balance de dicha ciudad de la inmigración y emigración interurbana (Tabla 1).

El siguiente, se ha dedicado a la elaboración y análisis de la red, formada por las ciudades, que constituyen los nodos, y por el número de migrantes y sus ciudades de origen y destino, representados por las vectores (aristas o conexiones) entre los nodos. De la red se ha buscado medir su estructura global y local a través de un conjunto de indicadores, que estiman la centralización y dispersión, la función de los nodos en la red y su agrupamiento (clúster). Los cálculos y gráficos se han elaborado con los programas ORA-NET SCENES 3.0 (Carley, 2017) y Cytoscape (Shannon et al., 2003).

En general, las medidas están construidas a partir de diferentes tipos de relaciones que se pueden establecer entre los nodos o grupos de nodos y 
TABLA 1. ORGANIZACIÓN DE LOS DATOS.

\begin{tabular}{|c|c|c|c|c|}
\hline & 1 & 2 & $\mathrm{n}$ & \\
\hline 1 & 0 & $\mathrm{~s}_{12}$ & $\mathrm{~s}_{1 \mathrm{n}}$ & $\sum_{1}^{\mathrm{n}} \mathrm{s}$ \\
\hline 2 & $\mathrm{~s}_{21}$ & 0 & & \\
\hline $\mathrm{m}$ & $\mathrm{S}_{\mathrm{m} 1}$ & & 0 & $\sum_{\mathrm{j}}^{\mathrm{n}} \mathrm{s}$ \\
\hline & $\sum_{1}^{\mathrm{m}} \mathrm{s}$ & & $\sum_{\mathrm{i}}^{\mathrm{m}} \mathrm{s}$ & $\sum_{\mathrm{ij}}^{\mathrm{nm}} \mathrm{s}$ \\
\hline
\end{tabular}

$\left(\mathrm{s}_{i j}\right) i, j=1, \ldots \mathrm{n}$; s: contingente de migrantes de la ciudad $i$ en que residen en la ciudad $j ; n=m, 44$ ciudades en 1987-1992 y 46 ciudades en 1997-2002).

aristas y los valores de sus atributos. De las medidas disponibles para describir las redes, que rebasan el centenar, se han seleccionado aquellas que, a priori, son más congruentes con la naturaleza del fenómeno aquí tratado, es decir, con una red bilateral, direccionada y con información espacial, en la que es posible la relación directa de un nodo con cualquier otro de la red, sin utilizar otros intermedios.

Entre los descriptores sencillos, figuran diversos cocientes entre el número de nodos y aristas y la distribución de aristas entrantes, salientes y total entre los nodos. Los agrupamientos se han identificado mediante el procedimiento jerárquico de
Newman. Las medidas más complejas corresponden a los indicadores de centralización siguientes: (Tabla 2) (Hanneman y Riddle, 2005).

Finalmente, el tercero está compuesto por los procedimientos aplicados para identificar y caracterizar los patrones geográficos de la red de migrantes a partir de distancia media recorrida por los emigrantes e inmigrantes de cada ciudad a las restantes, estimada en línea recta. Para su obtención se han utilizado los vectores origen-destino de los migrantes: se ha calculado la distancia media ponderada $\left(D_{i}\right)$ recorrida por los desplazados de cada ciudad al resto de ciudades $\left(\mathrm{s}_{\mathrm{ij}}\right)$ : contingente de migrantes de la ciudad $i$ en la ciudad $j ; d_{i j}$ : distancia geodésica entre la ciudad $i$ y la $j$ )

$$
\mathrm{D}_{i} \sum_{\mathrm{ij}}^{\mathrm{n}} \mathrm{s}_{\mathrm{ij}} \mathrm{d}_{\mathrm{ij}} / \sum_{\mathrm{ij}}^{\mathrm{n}} \mathrm{s}_{\mathrm{ij}}
$$

De modo análogo, para valorar los resultados empíricos, se ha obtenido la distancia media teórica de cada ciudad a las restantes.

La visualización es un recurso muy útil que facilita la interpretación de información relacional, como la estudiada aquí (Rae, 2009). Por tanto, los mapas y gráficos elaborados constituyen parte esencial del trabajo. El análisis espacial y los mapas se han llevado a cabo con el programa ArcGIS 10.5 (ESRI, s.f.). 


\section{TABLA 2. INDICADORES DE CENTRALIZACIÓN Y AGRUPAMIENTOS (CLUSTERING)}

\section{Medida/Formulación}

Centralidad eigenvector: vector combinado de todos los valores escalados $\mathrm{V}_{\mathrm{k}}$ de una red.

Centralidad hub $=$ centralidad eigenvector de la red $A^{*} A$

(Mín. = 0; Máx. = 1)
Centralización eigenvector de la red $=\mathrm{c} /(\mathrm{N}-1)$

Centralidad In-Degree = suma de columna (A) / $\mathrm{V}^{*}$ filas (A)

(Mín. = 0; Máx. = 1)

Centralidad Out-Degree $=$ suma de fila (A) $/ V^{*}$ columnas $(A)$

(Mín. = 0; Máx. =1)

\section{Descripción}

Sean: $\mathrm{A}$ una red unimodal de $\mathrm{N}$ nodos; $\mathrm{K}$ el número de componentes de la red $\mathrm{A}$; $\mathrm{N}_{\mathrm{k}}$ nodos del componente $\mathrm{kth} ; \mathrm{V}_{\mathrm{k}}$ eigenvector dominante de la subred inducida por los nodos $\mathrm{N}_{\mathrm{k}}$. Se escalan los valores $\mathrm{V}_{\mathrm{k}}$ multiplicándolos por $\mathrm{N}_{\mathrm{k}} / \mathrm{N}$ (Bonacich, 1972)

Generalización de la centralidad eigenvector (Kleinberg, 1998) c: valor del vector v; v: valor del vector de igencentralidad de la red; N: número de nodos

A: red de entrada; V: valor máximo de las aristas (link)

La centralidad total-degree es la suma normalizada de la centralidad lin-Degre y Out-Degree

k-core

Identificación de agrupamientos (clúster), por el método de Newman
Subred en la que cada nodo posee un centralidad total-degree igual o mayor que $k$ (Seidman, 1983)

Procedimiento jerárquico de agrupamiento de nodos basado en la similitud de su estructura de los flujos (modularidad) (Clauset et al., 2004 ; Newman, 2003)

Fuente: Carley, 2017 


\section{Características, dinámica, estructura y patrones espaciales del modelo de migraciones residenciales interurbanas}

En el período estudiado, las migraciones residenciales interurbanas de las ciudades más grandes (mayores de 50.000 habitantes) han incrementado ligeramente su peso en relación con las migraciones residenciales de los asentamientos por debajo de este umbral y, además, han mantenido el ritmo durante la década del cambio de los siglos XX al XXI, frente al retroceso de la movilidad residencial registrado en el resto del país durante el mismo período. La estructura de los flujos residenciales interurbanos es muy polarizada y apenas ha cambiado en este decenio, pero sí se han producido cambios locales en el origen, destino y magnitud de los flujos. Los aspectos más importantes de esta dinámica se describen a continuación.

\section{IMPORTANCIA CRECIENTE DE LAS CIUDADES MÁS GRANDES}

Entre 1992 y 2002 el peso demográfico de las ciudades mayores de 50.000 habitantes se incrementó, en grado considerable, respecto de la población total de Chile debido a las aportaciones de los flujos migratorios procedentes de asentamientos de menor tamaño con destino a aquellas ciudades.

Por otra parte, las migraciones residenciales habidas exclusivamente entre estas ciudades aumentaron en 95.029 personas, lo que ha subido la proporción de la movilidad residencial de este grupo de ciudades respecto de la total (intercomunal) del país, pues ha pasado del $24,5 \%$ al $28,7 \%$ entre 1992 y 2002 (Tabla 3). Este dinamismo, aunque sea paralelo al crecimiento de la población total, contrasta fuertemente con el descenso de la movilidad residencial intercomunal del conjunto del Chile, que retrocedió un 3,5\% en el mismo decenio. Estos datos indican que, durante esta década, las migraciones residenciales de las ciudades no se intensificaron pero sostuvieron el mismo pulso frente a la desaceleración del promedio del país. 
TABLA 3. PRINCIPALES MAGNITUDES DE LAS MIGRACIONES INTERURBANAS EN CHILE, 1992 Y 2002.

\section{7-1992}

1997-2002

Número de ciudades (> 50.000 habitantes)

44

46

Población total (de la muestra de ciudades)

7.853.382

9.381 .092

$\%$ de la población de Chile

58,8

62,1

Migrantes

565.580

660.609

$\%$ de la población de las ciudades

7,2

7,0

Número de ciudades con balance neto (inmigrantes-emigrantes) positivo

28

Número de ciudades que han cambiado el signo de su balance en el decenio: de positivo a negativo

Número de ciudades que han cambiado el signo de su balance en el decenio: de negativo a positivo

Número de relaciones bilaterales

1.872

Promedio de personas/relación bilateral

302

324

Fuente: INE, Censos de población y vivienda, 1992 y 2002. Elaboración propia.

El volumen de los flujos de la emigración e inmigración de las ciudades muestra, en ambos períodos, una relación lineal y una correlación muy alta con la población total ( $\mathrm{R}^{2}$ en torno a 0,9 , en todos los casos). No obstante, en el decenio que media entre los censos, se ha producido un cambio relevante en esta relación: las ciudades más grandes concentran más inmigrantes y emigrantes en relación con su población total, como indica el aumento de las pendientes de las funciones potenciales que ajustan la población total y los flujos migratorios (1992: Inmigrantes: $y=0,5628 x^{0,852} R^{2}=$ 0,92 Emigrantes: $y=0,316 x^{0,895} R^{2}=0,94 ; 2002$ : Inmigrantes: $y=0,0842 \times{ }^{1,005} R^{2}=0,89$ Emigrantes: $y=0,28 x^{0,909} R^{2}=0,89$ ) (Figura 1). 


\section{FIGURA 1. RELACIÓN ENTRE EL TAMAÑO DEMOGRÁFICO DE LAS CIUDADES Y LOS FLUJOS MIGRATORIOS INTERURBANOS: 1987-1992 (IZOUIERDA) Y 1997-2002 (DERECHA).}
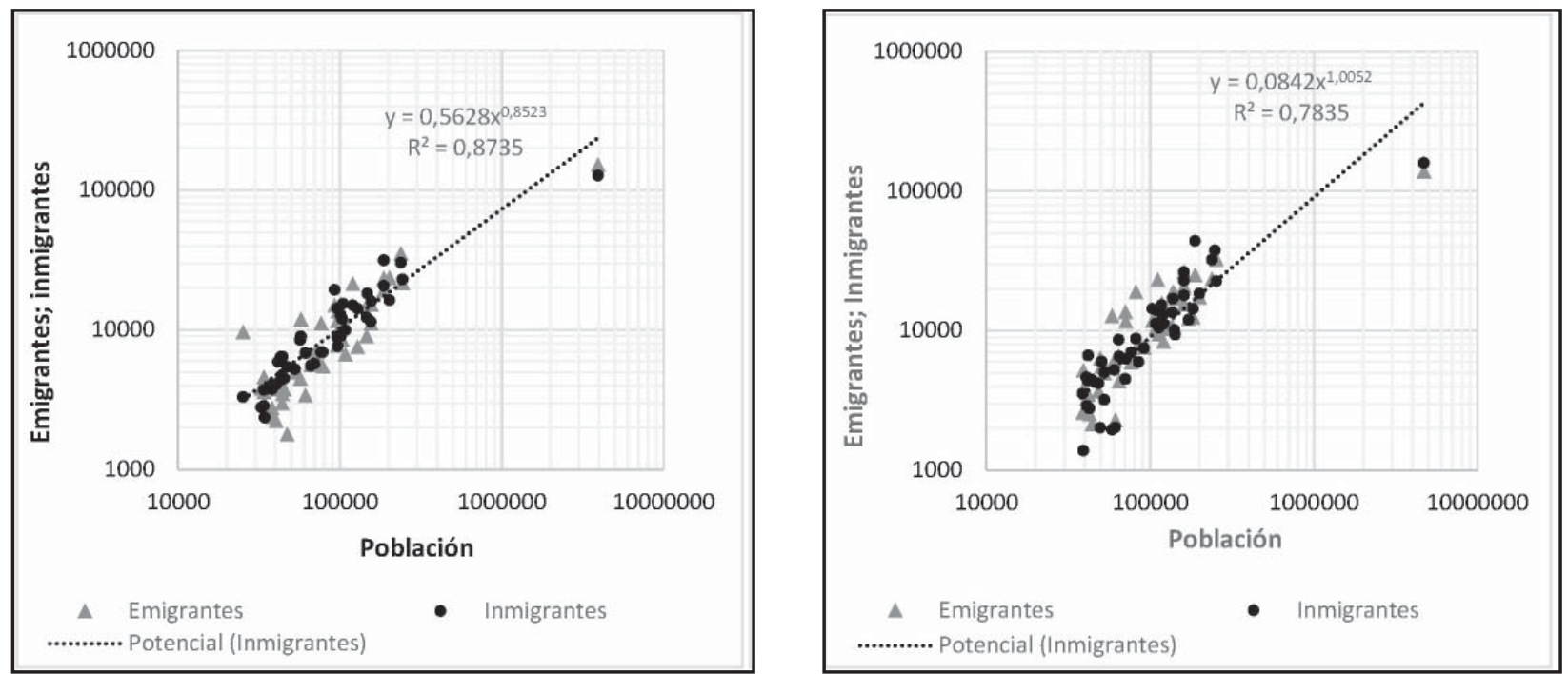

Fuente: INE, Censos de población y vivienda, 1992 y 2002. Elaboración propia.

Las características de los migrantes del período 1997-2002 revelan algunos aspectos específicos de los migrantes interurbanos comparados con los no migrantes, como la mayor movilidad de las mujeres, la gran prevalencia de los migrantes con formación universitaria respecto de los migrantes de los demás niveles educativos y el predominio de los migrantes jóvenes. Estos rasgos sugieren que el grueso de la movilidad interurbana tiene carácter laboral, y está compuesta por personas jóvenes, bien formadas, que se desplazan a otra ciudad para mejorar sus condiciones de trabajo, pues casi la mitad de los migrantes tienen alguna ocupación (Tabla 4). El predominio laboral no excluye otros motivos de migración, relacionados con las características geográficas de los lugares de destino -proximidad, clima, calidad ambiental-, con circunstancias personales de los migrantes o con otros motivos (por ejemplo, los efectos de desastres que fuerzan al cambio de residencia: grandes incendios -Valparaíso-, erupciones volcánicas, tsunamis, etc.). 


\section{TABLA 4. ALGUNAS CARACTERÍSTICAS DE LOS NO MIGRANTES Y MIGRANTES ENTRE CIUDADES EN $2002(\%)$.}

$\begin{array}{lrr}\text { Características } & \text { No migrantes (\%) } & \text { Migrantes (\%) } \\ \text { Sexo } & & \\ \text { Mujeres } & 47,75 & \mathbf{5 2 , 0 5} \\ \text { Hombres } & 52,25 & 47,95 \\ \text { Edad } & & \\ \text { De 0 a 19 años } & 28,55 & 27,14 \\ \text { De 20 a 24 años } & 40,85 & \mathbf{5 4 , 5 2} \\ \text { De 45 a 64 años } & 21,64 & 13,83 \\ \text { De 65 y más años } & 8,96 & 4,50 \\ \text { Nivel educativo } & & \\ \text { Básica } & 39,64 & 28,60 \\ \text { Media } & 29,98 & 29,07 \\ \text { Técnica } & 17,35 & 19,14 \\ \text { Universitaria } & 11,27 & \mathbf{2 1 , 8 4} \\ \text { Situación laboral } & & \\ \text { Ocupados } & 43,29 & \mathbf{9 , 9} \\ \text { Desocupados } & 9,56 & \mathbf{4 4 , 5 8} \\ \text { Dependientes } & 47,15 & \end{array}$

Fuente: INE, Censos de población y vivienda, 1992 y 2002. Elaboración propia.

Nota: La aplicación del test $\chi^{2}$ confirma la existencia de diferencias significativas en todas las variables, al menos al $95 \%$. No obstante, hay que notar que los resultados corresponden a números muy grandes, por lo que deben tomarse con cautela.

\section{ESTABILIDAD DE LA ESTRUCTURA GENERAL Y ELEVADA MOVILIDAD LOCAL}

Durante el decenio 1992-2002 la red de migrantes interurbanos de las ciudades mayores de 50.000 habitantes creció en tamaño y registró múltiples cambios locales, aunque su estructura agregada se mantuvo bastante estable. La densidad de la red es muy elevada en ambos periodos estudiados, de tal forma que el 99\% de las ciudades mantienen flujos migratorios bilaterales con todas las demás. Sin embargo, si la densidad se calcula con los flujos por encima de la mediana (47 personas), su valor se reduce a menos de la mitad: $48,7 \%$.

Una de las propiedades más destacables de la estructura global de la red migratoria es su elevado grado de centralización, solo levemente más bajo en 2002 que en 1992.

La medida de centralización eigenvector de la red, que estima el modo en que se distribuyen y conectan todos los flujos de la red, ha permanecido prácticamente estacionaria entre 1992 y $2002(0,76$ y 0,75 , respectivamente), lo que indica la similitud estructural de ambos modelos.

La notable estabilidad de la estructura es compatible -y producida- con múltiples cambios en la 


\section{FIGURA 2. FLUJOS Y SALDOS MIGRATORIOS 1987-1992 (IZQUIERDA) Y 1997-2002 (DERECHA).}

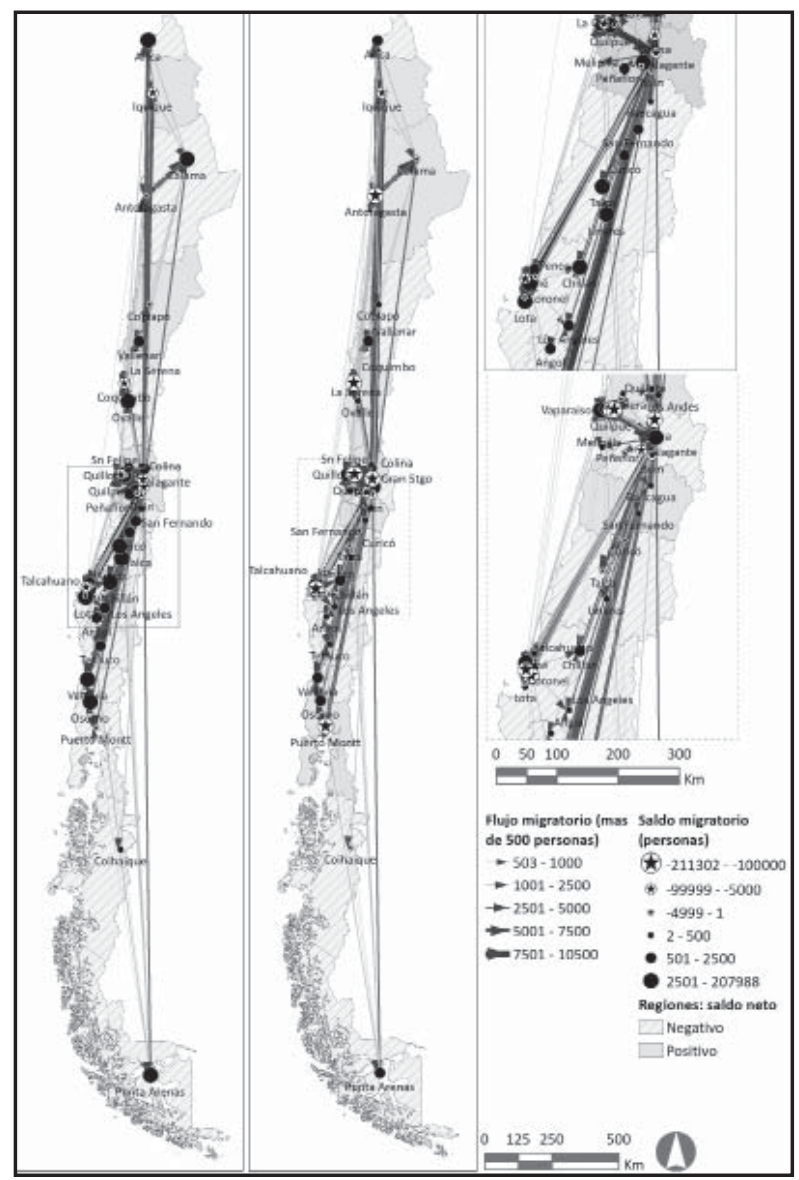

Fuente: INE, Censos de población y vivienda, 1992 y 2002. Elaboración propia. dirección y magnitud de los flujos de migrantes y del reparto desigual de los saldos migratorios entre las ciudades. Todos los indicadores muestran que, entre los dos censos, ha crecido la concentración de los inmigrantes en algunos destinos y se ha dispersado levemente el origen de los emigrantes.

En los mapas de la Figura 2 se representa el origen, destino y magnitud de los flujos migratorios, así como el signo y volumen de los saldos migratorios para los períodos estudiados. En ambos mapas se observa, por una parte, la concentración en Santiago de los flujos entrantes y salientes más voluminosos y, por otra, la mayor densidad de conexiones bilaterales en las regiones centrales del país, acorde con la estructura más compleja de la red urbana de estas regiones.

En la Tabla 5 se recogen datos que resumen la magnitud de algunos cambios significativos mencionados en las principales ciudades.

En primer lugar, hay que destacar las diferentes proporciones de inmigrantes y emigrantes que concentran las ciudades con mayores y menores saldos migratorios en 1987-1992 y 1997-2002. Si, en el primer período, las ciudades con los cinco saldos migratorios mayores suponían menos de la mitad del total de los saldos positivos (42,7\%), este mismo indicador ascendía al 84,7\% en el segundo quinquenio, es decir, refleja la fuerte concentración de los inmigrantes, principalmente en el Gran Santiago y, en menor medida, en las otras ciudades 
más grandes. En cambio, como ya se ha indicado, los saldos negativos están menos concentrados en 2002 que en 1992.

Otro aspecto relevante de los datos se encuentra en el cambio de signo de los saldos migratorios que han registrado algunas ciudades entre ambos censos. Tal vez el caso más destacable es el del Gran Santiago, que pasa de tener saldo migratorio negativo en el primer período a positivo en el segundo, igual que Copiapó, Viña del Mar, Talcahuano y otras ciudades. En sentido contrario, Quilpué, Calama, Talca, Curicó, Peñaflor, entre otras ciudades, oscilan de saldos positivos a negativos.

El balance del saldo migratorio, positivo o negativo, se debe a diferentes combinaciones de los contingentes de personas que llegan o salen de cada ciudad. Como se ha indicado, el volumen migratorio entre las ciudades del estudio ha crecido algo más de 95.000 personas entre 1992 y 2002, pero no se ha distribuido de forma homogénea entre las mismas.

Los inmigrantes han aumentado considerablemente, durante este período, en el Gran Santiago, Concepción, Talcahuano, Valparaíso e Iquique; al contrario, han descendido de forma muy acusada en
Quilpué, Peñaflor, Buin y La Calera. Por otro lado, el número de emigrantes ha crecido notablemente en Antofagasta, Villa Alemana, Puerto Montt y La Serena.

El crecimiento de la movilidad residencial ha ido acompañado de variaciones en la dirección y volumen de los flujos, que son síntomas y causas, al mismo tiempo, de cambios en el sistema de relaciones entre estas ciudades. En el mapa de la Figura 3 se representan los principales cambios de los flujos origen-destino habidos entre los quinquenios analizados. En el mismo destaca, por una parte, que las ciudades en las que descienden los inmigrantes se localizan en las regiones centrales y, además, se localizan cerca de otras grandes ciudades. Por otra, sobresale el crecimiento de los flujos de inmigrantes hacia el Gran Santiago desde ciudades de todo el territorio así como el decrecimiento de las corrientes que parten desde Gran Santiago a otras ciudades. También se pueden identificar agrupamientos de ciudades cuyos cambios están mutuamente relacionados, como el formado por Valparaíso, Viña del Mar, Quilpué y Villa Alemana. En otros casos, los cambios aparecen espacialmente encadenados, como en Puerto Montt, Valdivia, Osorno y Temuco. 
TABLA 5. MIGRANTES Y SALDO MIGRATORIO 1987-1992 Y 1997-2002 (CINCO CIUDADES CON SALDOS MAYORES; CINCO CIUDADES CON SALDOS MENORES).

\begin{tabular}{|c|c|c|c|c|c|c|c|}
\hline \multicolumn{4}{|c|}{ 1987-1992 } & \multicolumn{4}{|c|}{ 1997-2002 } \\
\hline Ciudad & $\begin{array}{r}\text { Emigrantes } \\
\text { (Miles) }\end{array}$ & $\begin{array}{r}\text { Inmigrantes } \\
\text { (Miles) }\end{array}$ & $\begin{array}{r}\text { Saldo } \\
\text { (Miles) }\end{array}$ & Ciudad & $\begin{array}{r}\text { Emigrantes } \\
\text { (Miles) }\end{array}$ & $\begin{array}{r}\text { Inmigrantes } \\
\text { (Miles) }\end{array}$ & $\begin{array}{r}\text { Saldo } \\
\text { (Miles) }\end{array}$ \\
\hline Concepción & 19,1 & 31,5 & 12,3 & G. Santiago & 137,4 & 159,0 & 21,6 \\
\hline Chillán & 7,5 & 14,2 & 6,7 & Concepción & 24,8 & 43,9 & 19,1 \\
\hline Quilpué & 15,1 & 19,4 & 4,3 & Valparaíso & 23,4 & 32,2 & 8,8 \\
\hline Ovalle & 4,5 & 8,5 & 4,0 & Talcahuano & 20,7 & 26,2 & 5,5 \\
\hline Lota & 1,8 & 5,5 & 3,7 & $\begin{array}{r}\text { Viña del } \\
\text { Mar }\end{array}$ & 33,9 & 37,6 & 3,7 \\
\hline 5 ciudades & 48,0 & 79,1 & 31,0 & 5 ciudades & 240,2 & 298,9 & 58,7 \\
\hline$\%$ del total & 8,5 & 14,0 & $42,7^{*}$ & $\%$ del total & 36,4 & 45,2 & $84,7^{*}$ \\
\hline G. Santiago & 152,2 & 127,4 & $-24,8$ & Colina & 12,7 & 1,9 & $-10,8$ \\
\hline Talcahuano & 23,8 & 16,3 & $-7,5$ & $\begin{array}{r}\text { Villa } \\
\text { Alemana }\end{array}$ & 18,9 & 8,7 & $-10,2$ \\
\hline Iquique & 21,4 & 15,0 & $-6,4$ & Antofagasta & 32,2 & 22,6 & $-9,6$ \\
\hline Colina & 9,6 & 3,3 & $-6,3$ & Quilpué & 23,0 & 13,7 & $-9,3$ \\
\hline Coquimbo & 13,6 & 7,6 & $-6,0$ & $\begin{array}{r}\text { S. Pedro } \\
\text { de Paz }\end{array}$ & 13,6 & 4,5 & $-9,1$ \\
\hline 5 ciudades & 220,6 & 169,6 & $-51,0$ & 5 ciudades & 100,4 & 51,4 & $-49,0$ \\
\hline$\%$ del total & 39,0 & 30,0 & $70,5^{*}$ & $\%$ del total & 15,2 & 7,8 & $53,9 *$ \\
\hline
\end{tabular}

Fuente: INE. Censos de población y vivienda, 1992 y 2002. Elaboración propia.

* Porcentaje del total de saldos positivos o negativos 


\section{FIGURA 3. CAMBIOS EN LOS FLUJOS Y MIGRATORIOS INTERURBANOS ENTRE EL OUINQUENIO 1987-1992 Y EL OUINQUENIO 1997-2002.}

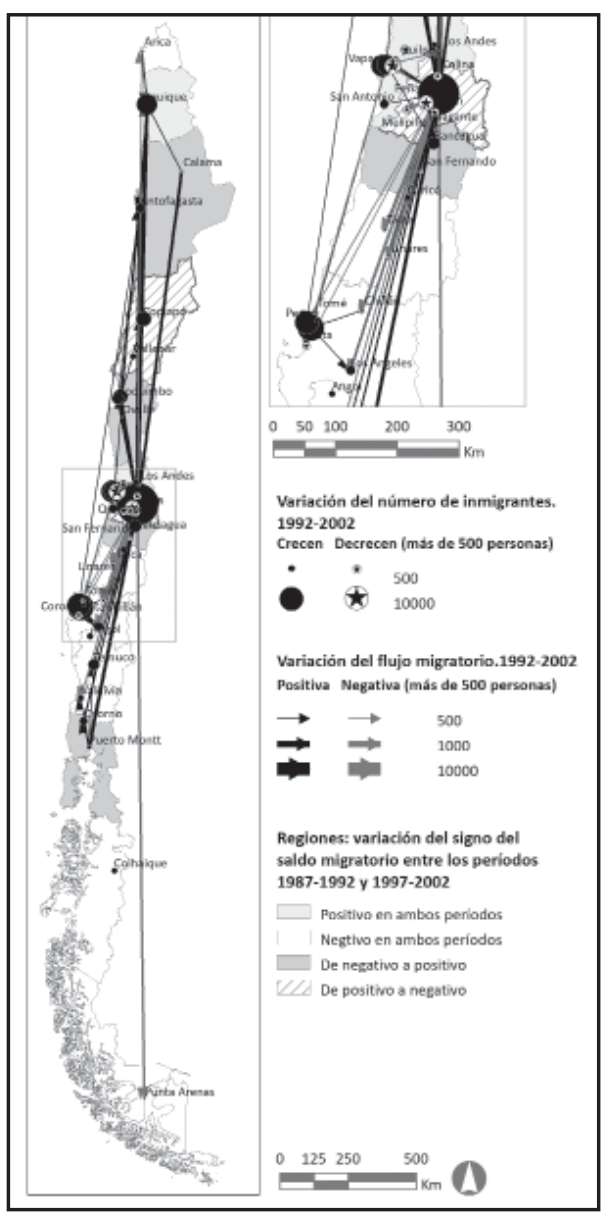

Fuente: INE, Censos de población y vivienda, 1992 y 2002. Elaboración propia.
Conviene recordar que los datos manejados se refieren, exclusivamente, a los cambios residenciales habidos entre las ciudades mayores de 50.000 habitantes, por lo que el número de migrantes y los valores de los saldos migratorios de las ciudades pueden diferir, en cierto grado, de los obtenidos cuando se incluyen todos los asentamientos. Habida cuenta de esta precaución, los resultados expuestos muestran que la movilidad residencial, ha crecido, entre 1992 y 2002, el 16,8\%, valor más elevado que el incremento del promedio de la movilidad residencial intercomunal que fue del 15,7\% en el mismo período. Estos resultados están en línea con algunos cambios avanzados en la "hipótesis de la transición de la movilidad" (Zelinsky, 1971), en particular con el incremento de la proporción de la movilidad interurbana -residencial en este caso- frente a otros flujos migratorios, pero no se observa su intensificación en Chile en los quinquenios comparados.

Uno de los impactos de estos cambios afecta al crecimiento demográfico de las ciudades. La migración ha sido, tradicionalmente, un factor de crecimiento de la población de las ciudades y regiones chilenas (Martínez, 2002; Rodríguez y González, 2006). En las migraciones residenciales estudiadas, las ciudades beneficiarias, desde el punto de vista demográfico, han sido las más grandes (Santiago, Concepción, Viña del Mar, Valparaíso; Talcahuano); las pérdidas están repartidas de forma más homogénea, pero las de algunas ciudades, como Colina y Villa 
Alemana, suponen, cada una, 10\% de las pérdidas del conjunto de ciudades. En conjunto, se ha mantenido el grado de centralización de la capital del país respecto del resto de las ciudades, pues solo unas cuantas ciudades más grandes, principalmente las citadas arriba, han ganado peso total como focos atractores de migración residencial. En otras, en cambio, ha menguado su saldo positivo (Chillán, Talca) o se han hecho más grandes sus saldos negativos (Antofagasta, Coquimbo, La Serena, Puerto Montt).

Las migraciones residenciales evidencian que en la década estudiada se ha producido una consolidación de las áreas metropolitanas, pero no un descenso significativo de la polarización ejercida por la capital del país y por un reducido grupo de ciudades. A otra escala, el balance migratorio refleja también la dinámica del mercado de la vivienda, no solo en las áreas metropolitanas, sino también en ciudades turísticas.

\section{ORGANIZACIÓN Y PATRONES ESPACIALES DE LAS MIGRACIONES RESIDENCIALES EN 1997-2002}

La organización de la red de flujos migratorios interurbanos se puede describir por las partes que componen dicha red (subredes), por los agrupamientos de ciudades generados por la fuerte intensidad de sus flujos mutuos y por la función predominante que cada ciudad desempeña en el sistema de flujos migratorios. La proximidad espacial entre ciudades y otras circunstancias -relaciones personales, percepción de la calidad del espacio urbano y de los equipamientos, de la seguridad, etc.- dan lugar a patrones espaciales y funcionales de diverso alcance y configuración.

Una forma de visualizar algunas características fundamentales de la estructura de la red es la de la imagen de la Figura 4. La posición de cada ciudad en el plano indica el grado de atracción-repulsión recíprocas, estimado por la intensidad de sus conexiones: la atracción es más intensa si los flujos mutuos son mayores, y menor si un flujo es elevado y otro débil. El tamaño de los símbolos representa su valor eigenvector, es decir, su importancia en el conjunto de la red en relación con sus conexiones con otras ciudades y de estas con otras terceras hasta completar la red. Este indicador también revela cuales son las ciudades que forman el núcleo (core) de la red, así como las subredes, más pequeñas y menos densas que este. Finalmente, las líneas y su grosor indican los valores de los flujos entre las ciudades de origen y destino con más de 250 migrantes.

La organización de la red de migrantes interurbanos combina varios niveles de estructuras y subestructuras, como se aprecia en la imagen. En conjunto, se trata de un modelo monocéntrico y muy polarizado, con forma de estrella, es decir, con conexiones directas entre el foco principal, Santiago, y los secundarios, las capitales regionales, que a 


\section{FIGURA 4. CENTRALIDAD EIGENVECTOR, K-CORE Y FLUJOS MIGRATORIOS RESIDENCIALES INTERURBANOS MAYORES DE 250 PERSONAS. 2002.}

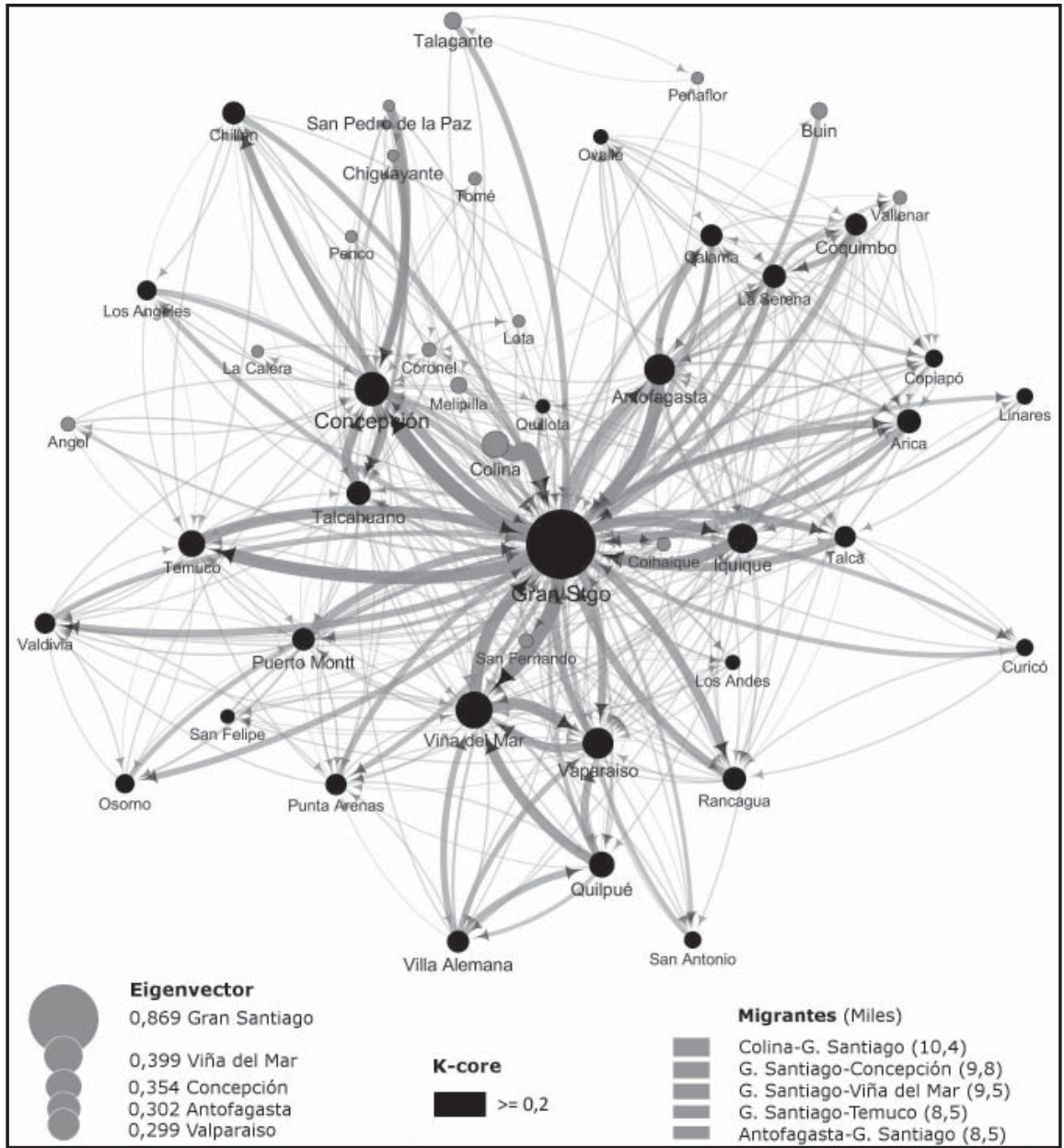

Fuente: INE. Censos de población y vivienda, 1992 y 2002. Elaboración propia. 
su vez actúan como centros de atracción de otras ciudades de su región.

El núcleo de la red (k-core: 0,177) está constituido por las principales ciudades y, en conjunto, es un componente gigante, bastante permanente en ambos quinquenios, que da gran estabilidad estructural a la red; el resto de las partes tienen un tamaño bastante menor que este núcleo. Algunas ciudades actúan como hub del sistema, pues sus emigrantes se dirigen a ciudades a las que también llegan emigrantes de otras varias ciudades (en 2002, están una desviación típica por encima de la media: Santiago, Viña del Mar, Antofagasta, Iquique, Colina, Valparaíso). Otras terceras, son emisoras o receptoras netas de migrantes (Tabla 5).

Los agrupamientos de ciudades, caracterizados por sus fuertes conexiones mutuas, adquieren una importancia fundamental en la organización del conjunto de la red de flujos migratorios. Como se observa en la Figura 4, los clústeres se componen de agregados de ciudades de diferentes tamaños. En la Tabla 6 se recogen algunas características de los cuatro grupos de ciudades delimitados mediante la aplicación del método de Newman, que utiliza como criterio de agrupamiento la maximización de la modularidad (medida de la tendencia de las redes a funcionar en módulos); los grupos se representan en el mapa de la Figura 5.
Estos agrupamientos muestran diferencias destacables en su organización espacial y en sus propiedades funcionales. El grupo más grande, el 1, está compuesto por 20 ciudades que se localizan en una franja que se extiende por el centro y sur del país, desde Colina hasta Coihaique. Las ciudades del grupo 2 también adoptan una disposición longitudinal, desde Arica, al norte, hasta Ovalle, en el centro. En ambos casos, la ruta 5 constituye el principal eje vertebrador de los sistemas urbanos. El grupo 4, posee una de las configuraciones más compactas, al igual que la mayor parte de las ciudades del grupo 3. La ciudad de Punta Arenas se incluye en este último grupo (Valparaíso-Viña del Mar), aunque sus flujos migratorios bilaterales más grandes los tiene con Santiago. Sin embargo, la suma de las relaciones migratorias con las ciudades de este grupo 3 -que, a su vez, están muy interconectadas- son mayores que las que mantiene con cualquier otro grupo. De ahí su inclusión en este clúster, lo que incrementa considerablemente el recorrido medio de los migrantes de este grupo y reduce su compacidad espacial.

Los clústeres identificados también contrastan por su grado de cohesión interna. El grupo 1 se caracteriza por la considerable intensidad de sus conexiones migratorias recíprocas, frente a los otros tres, cuyo grado de apertura es considerablemente mayor, como indican sus respectivos valores 
TABLA 6. PRINCIPALES CARACTERÍSTICAS DE LOS CLÚSTERES. 2002

\begin{tabular}{|c|c|c|c|c|c|c|c|c|c|c|}
\hline 을 & $\begin{array}{l}\frac{\mathscr{d}}{0} \\
\frac{\pi}{0} \\
\text { 음 }\end{array}$ & 응 & 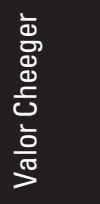 & 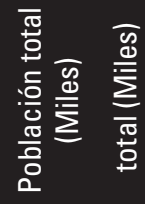 & 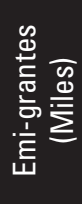 & 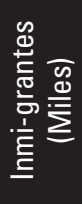 & $\begin{array}{l}\frac{a}{0} \\
\frac{d}{0} \\
\frac{\pi}{0} \\
\frac{\partial}{0}\end{array}$ & $\frac{\widehat{a}}{\frac{0}{0}}$ & 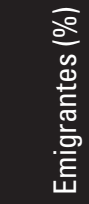 & 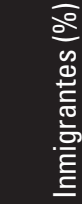 \\
\hline 1 & 20 & 0,456 & 50,9 & 6.503 & 296 & 305 & 43,5 & 69,3 & 46,2 & 44,9 \\
\hline 2 & 9 & 0,802 & 73,0 & 1.186 & 147 & 132 & 19,6 & 12,7 & 20,0 & 22,3 \\
\hline 3 & 9 & 0,802 & 73,0 & 991 & 130 & 126 & 19,6 & 10,6 & 19,0 & 19,7 \\
\hline 4 & 8 & 0,826 & 73,2 & 700 & 87 & 97 & 17,4 & 7,5 & 14,8 & 13,2 \\
\hline Total & 46 & & 73,0 & 9.380 & 660 & 660 & 100,0 & 100,0 & 100,0 & 100,0 \\
\hline
\end{tabular}

Valor Cheeguer: ratio entre el número de conexiones externas de un grupo y su tamaño número de nodos (ciudades). El índice E - I mide el grado de cohesión interna de cada agrupamiento; E - I = (conexiones externas-conexiones internas) / total conexiones. (-1 $\leq \mathrm{E} I \leq 1)$.

Fuente: INE. Censos de población y vivienda, 1992 y 2002. Elaboración propia.

Cheeguer e índices E-I. El examen de los flujos migratorios revela que los agrupamientos se forman, sobre todo, por la fuerza polarizadora ejercida por las grandes entidades urbanas funcionales, especialmente Santiago (grupo 1) (Schiappacasse, Contreras y Fuensalida, 2001) y, en menor medida, Valparaíso-Viña del Mar y Concepción-Talcahuano (grupos 3 y 4 respectivamente). 


\section{FIGURA 5. AGRUPAMIENTOS DE CIUDADES SEGÚN LA INTENSIDAD DE SUS CONEXIONES MIGRATORIAS. 2002.}

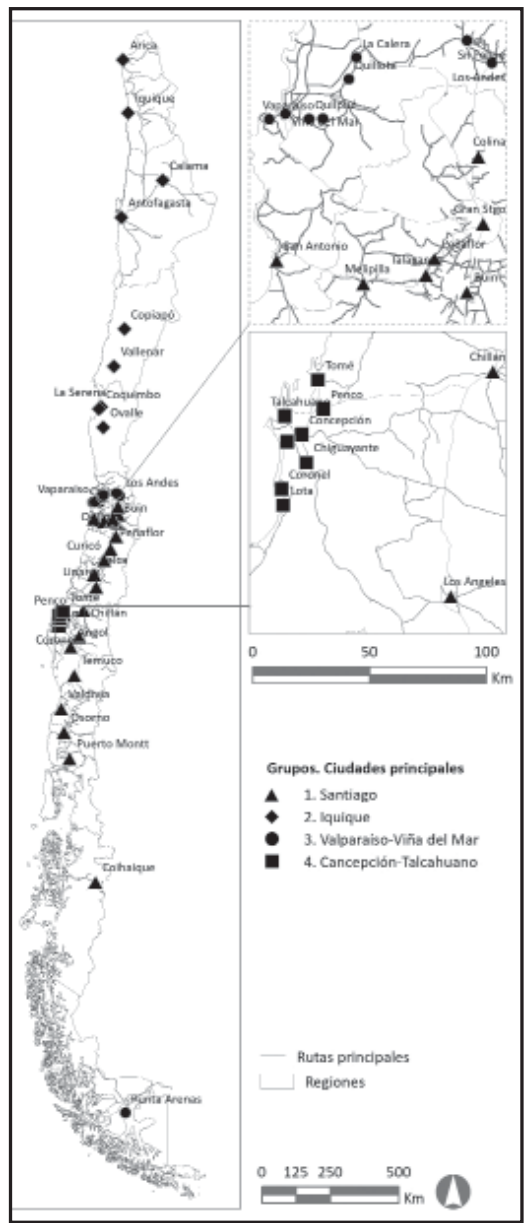

Fuente: INE, Censos de población y vivienda, 1992 y 2002. Elaboración propia.
La intensidad de los flujos migratorios depende, en gran medida, de la cercanía o lejanía de las ciudades, pues esta influye en los costes de traslado y, más aún, en la percepción y conocimiento que los emigrantes tienen de las oportunidades y características de las ciudades de destino. El análisis de la distribución de las distancias recorridas por los migrantes pone de manifiesto la gran influencia que la cercanía entre las ciudades ejerce en cuanto al origen y destino de los migrantes y en lo relativo a la formación de agregados de ciudades con intensos flujos migratorios mutuos.

La distribución de los flujos migratorios según su tamaño sigue una ley potencial: la mayor parte de los flujos son de pequeño volumen, mientras que son pocos los flujos muy cuantiosos, aunque suponen peso elevado respecto del total de los migrantes. En el gráfico de la Figura 6 se representa a proporción de migrantes según intervalos de distancia de los desplazamientos de los migrantes en los quinquenios 1987-1992 y 1997-2002. Ambas distribuciones presentan diferencias significativas en los tramos de distancia intermedios y largos (de 101 a $250 \mathrm{~km}$, de 251 a $500 \mathrm{~km}$ y más de 500 $\mathrm{km}$ ): en el primer quinquenio tienen mayor peso los recorridos más largos y en el segundo los intermedios, lo que se traduce en el descenso de la distancia promedio de los flujos migratorios de 526 $\mathrm{km}$ a $510 \mathrm{~km}$ en el primer y segundo quinquenios, respectivamente. 


\section{FIGURA 6. DISTRIBUCIÓN DE LOS MIGRANTES POR INTERVALOS DE DISTANCIA RECORRIDA. 1987-1992 Y 1997- 2002.}

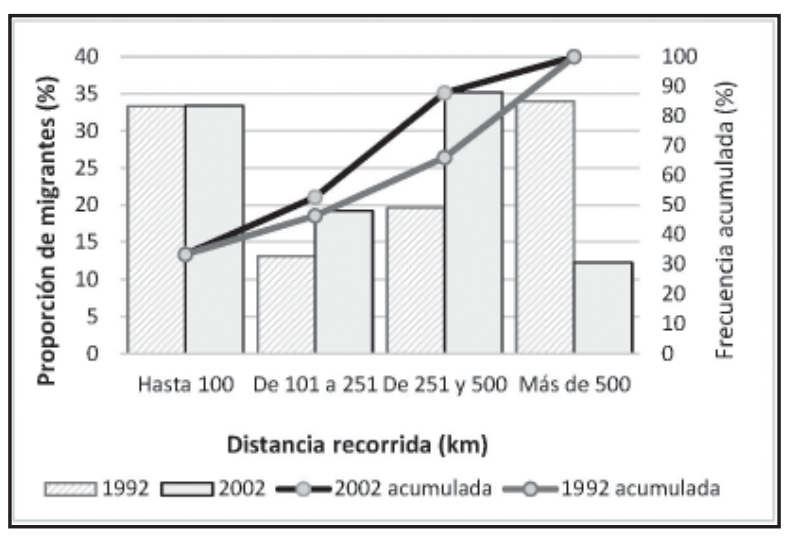

Fuente: INE. Censos de población y vivienda, 1992 y 2002. Elaboración propia.

Para valorar estos datos, es necesario tener en cuenta la estructura de la distribución espacial de las ciudades, que en su conjunto están separadas por una distancia promedio de $690 \mathrm{~km}$. Si se consideran los habitantes de cada ciudad, esta distancia se rebaja a $605 \mathrm{~km}$, debido al gran peso de la población del área central del país. En consecuencia, es lógico que los migrantes de las ciudades más excéntricas, del norte y sur del país, tengan recorridos mayores que los de las regiones del centro. Los datos empíricos muestran que las trayectorias de los flujos migratorios son muy eficientes ya que, en casi todos los casos, están por debajo de la distancia teórica que separa a cada ciudad de las demás. Ello se debe, básicamente, a la localización central de Santiago, que es el principal foco de atracción y salida de migrantes, y a la dispersión espacial de las capitales regionales, que son atractores secundarios: esta disposición de las ciudades y de la población minimiza los recorridos.

En resumen, la cercanía o lejanía entre las ciudades es una variable que modula la dirección y la magnitud de las migraciones residenciales, tanto por su influencia en el coste de los cambios de domicilio como por el mejor conocimiento que, generalmente, tienen los migrantes de las ciudades más cercanas a su ciudad de residencia. En el caso de Chile, el factor distancia está muy condicionado por el enorme tamaño demográfico, económico y simbólico de Santiago y su localización en el centro del país, variables que, hasta cierto punto, reducen la longitud de los flujos migratorios.

\section{Conclusiones}

En el presente trabajo se ha considerado que las migraciones residenciales interurbanas conforman una red única que representa las interacciones migratorias entre el conjunto de las ciudades del estudio. Este modelo es más adecuado para captar el entramado de flujos migratorios entre las ciudades 
que el análisis aislado de los flujos bilaterales entre cada ciudad.

La organización de las migraciones residenciales interurbanas de las ciudades mayores de 50.000 habitantes se puede caracterizar por tres aspectos interrelacionados: la elevada centralización de los inmigrantes, la gran estabilidad de la estructura de la red migratoria y su organización espaciofuncional jerarquizada en dos niveles principales. La persistencia estructural es compatible con movimientos y cambios en el volumen, origen y destino de los flujos migratorios a escala local, así como con la presencia de clústeres de ciudades y subestructuras locales de tamaño y alcance espacial moderados. El nodo principal de la red, Santiago, articula las migraciones residenciales a escala nacional, mientras que las capitales regionales y otras ciudades lo hacen a escala subnacional. Aunque la estructura está compuesta por múltiples centros de confluencia y salida de migrantes, la diferencia de volumen entre estos y Santiago es tan grande que dicha estructura se puede calificar de monocéntrica.

Por otra parte, los datos agregados de las características de los migrantes muestran que, en general, predominan los jóvenes, hombres y mujeres, con formación elevada: muy probablemente, el grueso de la corriente migratoria tiene un carácter laboral y está formada por individuos más que por familias.
Estos resultados sugieren que las actividades socioeconómicas impulsadas por la globalización han podido mantener la vitalidad en algunas ciudades capitales regionales, pero no han tenido la fuerza suficiente para crear puestos de trabajo que atraigan nuevos habitantes permanentes. En efecto, más de la mitad de las migraciones residenciales interurbanas son de corta distancia (menos de $300 \mathrm{~km}$ ) y configuran cuencas de atracción de inmigrantes, más o menos extensas, en torno a las principales ciudades.

Una de las razones que pueden explicar por qué no se han producido en este tiempo una la descentralización residencial más intensa, se debe a que las nuevas tecnologías de comunicación y las mejoras en infraestructuras facilitan las migraciones pendulares a larga distancia, que son dominantes en actividades mineras y de construcción (Rowe, 2014), y las estancias cortas, no recogidas en los datos censales. Por otro lado, persiste una gran concentración de actividades de servicios avanzados en las grandes ciudades, factor de atracción de inmigrantes.

Otras causas que cabe plantear se relacionan con las características de los migrantes y sus redes interpersonales. Es muy probable que los patrones espaciales de los migrantes difieran en función de su formación y edad. Para conocer esta posible variación es necesario desglosar los flujos migratorios según estas variables, lo que podría ser objeto de 
futuras investigaciones, actualizadas con los datos del censo de 2017. Los resultados obtenidos indican que en los períodos estudiados la gran influencia de la proximidad geográfica en los patrones de las migraciones residenciales e, indirectamente, en la creación de redes personales que se relacionan con las decisiones de los migrantes.

Si estudios futuros confirman que el modelado y análisis de redes son ventajosos para el conocimiento de los flujos migratorios interurbanos con un grado de aproximación aceptable, sería posible realizar previsiones, a corto y medio plazo, de los flujos migratorios entre ciudades, teniendo en cuenta las interacciones entre todos ellos e incluso, incorporar eventos inusuales. Este método podría constituir una alternativa o complemento a los modelos convencionales de interacción espacial. Resulta obvio que el conocimiento preciso de la dinámica y estructura espacial de las migraciones residenciales interurbanas tienen gran interés para múltiples propósitos y agentes, públicos y privados, concernidos por los diversos efectos de este tipo de migraciones.

\section{Referencias bibliográficas}

Arango, J. (2000). Explaining migration: A critical view. International Social Science Journal, 52(165), 283296. https://doi.org/10.1111/1468-2451.00259

Artal-Tur, A., Peri, G., y Requena-Silvente, F. (Eds.). (2014). The socio-economic impact of migration flows: effects on trade, remittances, output, and the labour market. Cham: Springer.

Barton, J.R., Gwynne, R.N., y Murray, W.E. (2007). Competition and co-operation in the semiperiphery: closer economic partnership and sectoral transformations in Chile and New Zealand. The Geographical Journal, 173(3), 224-241. https://doi. org/10.1111/j.1475-4959.2007.00237.x

Bonacich, P. (1972). Factoring and weighting approaches to status scores and clique identification. The Journal of Mathematical Sociology, 2(1), 113-120. https://doi.org/10.1080/0022250X.1972.9989806

Cadwallader, M.T. (1992). Migration and residential mobility: Macro and micro approaches. Madison, Wis.: University of Wisconsin Press.

Carley, K.M. (2018). ORA-NET SCENES (versión 6). [Software] Recuperado de http://www.casos. cs.cmu.edu/projects/ora/download.php.

Castles, S., Haas, H.d., y Miller, M.J. (2014). The age of migration: international population movements in the modern world (5a. ed.). New York: Guilford Press. 
Clauset, A., Newman, M.E.J., y Moore, C. (2004). Finding community structure in very large networks. Physical Review E, 70(6). https://doi.org/10.1103/ PhysRevE.70.066111

Di Filippo, A. y Bravo, R. (1977). Los centros nacionales de desarrollo y las migraciones internas en América Latina: un estudio de casos, Chile. EURE, 5(14), 67-101. Recuperado de http://www.eure.cl/ index.php/eure/article/view/897

Escolano, S. (2012). Dinámica reciente del sistema urbano chileno 1970-2002: integración a escala nacional. Boletín de la Asociación de Geógrafos Españoles, (59), 129-150. Recuperado de https:// www.age-geografia.es/ojs/index.php/bage/article/ view/1452

Escolano, S. y Ortiz, J. (2017). La localización de los servicios intensivos en conocimiento en Chile. En R. Sánchez, R. Hidalgo y F. Arenas (Eds.), Re-conociendo las geografías de América Latina y el Caribe (pp. 289-319). Santiago, Chile: Pontificia Universidad Católica de Chile.

ESRI. (s.f.). ArcGIS desktop (versión 10.5). Redlands (CA): Autor.

Gobierno de Chile. (2001). La inversión en infraestructuras 1990-1999 y su proyección 2000-2009. Santiago: MOP.

Hanneman, R.A. y Riddle, M. (2005). Introduction to social network methods. Riverside, CA: University Of California.

Henriquez, C. y Venegas, J. (2007). Contenido de importaciones en las exportaciones chilenas 1986-2005: análisis de insumo producto. Santiago: Banco Central.

Hidalgo, R., Mattos, C.d., y Arenas, F. (Eds.). (2009). Chile: del país urbano al país metropolitano. Santiago, Chile: Pontificia Universidad Católica de Chile.

Imilan, W., Garcés, A., y Margarit, D. (Eds.). (2014). Poblaciones en movimiento. Etnificación de la ciudad, redes e integración. Santiago, Chile: Universidad Alberto Hurtado.

Instituto Nacional de Estadísticas. (2009a). Compendio estadístico año 2008. Santiago: autor. Recuperado de http://www.ine.cl/canales/menu/publicaciones/compendio_estadistico/compendio_estadistico2008.php

Instituto Nacional de Estadísticas. (2009b). Parque de vehículos en circulación 2008. Santiago: autor. Recuperado de http://www.ine.cl/canales/chile_estadistico/estadisticas_economicas/transporte_y_ comunicaciones/parquevehiculos.php

Kleinberg, J.M. (1998, mayo). Decision algorithms for unsplittable flow and the half-disjoint paths problem. En J. Vitter (Chair), Symposium on Theory of Computing. Symposium conducted at the meeting of ACM. https://doi.org/10.1145/276698.276867

Lin, N., Cook, K.S., y Burt, R.S. (Eds.). (2001). Social capital: theory and research. New York: Aldine de Gruyter.

Luo, W. y MacEachren, A. M. (2014). Geo-social visual analytics. Journal of Spatial Information Science, (8), 27-66. https://doi.org/10.5311/JOSIS.2014.8.139 
Martínez, J. (2002). Ciudades de Chile, migración interna y redistribución de la población: algunas evidencias del período 1987-1992. Revista de Geografía Norte Grande, (29), 21-38.

Massey, D.S., Alarcón, R., Durand, J., y González, H. (Eds.). (1990). Return to Aztlan: the social process of international migration from western Mexico. Berkeley, Cal.: University of California Press.

Massey, D.S., Arango, J., Hugo, G., Kouaouci, A., PeIlegrino, A., y Taylor, J. E. (1998). Worlds in motion: understanding international migration at the end of the millenium. New York: Clarendon Press, Oxford University Press.

Mora, J. y Taylor, E. (2006). Determinants of migration, destination and sector choice: Disentangling individual, household and community effects. En Ç. Özden y M. Schiff (Eds.), International migration, remittances, and the brain drain (pp. 21-52). Washington D.C.: The World Bank, Palgrave Macmillan.

Newman, M.E.J. (2003). The structure and function of complex networks. SIAM Review, 45(2), 167-256. https://doi.org/10.1137/S003614450342480

Pumain, D. y Saint-Julien, T. (1990). Les migrations interurbaines. Mappemonde, 90(1), 33-34. Recuperado de https://www.mgm.fr/PUB/Mappemonde/ M190/m8.pdf

Putnam, R.D., Leonardi, R., y Nanetti, R. (1994). Making democracy work: civic traditions in modern Italy. Princeton, NJ: Princeton University Press.
Rae, A. (2009). From spatial interaction data to spatial interaction information? Geovisualisation and spatial structures of migration from the 2001 UK census. Computers, Environment and Urban Systems, 33(3), 161-178. https://doi.org/10.1016/j. compenvurbsys.2009.01.007

Rodríguez, J. y González, D. (2006). Redistribución de la población y migración interna en Chile: continuidad y cambio según los últimos cuatro censos nacionales de población y vivienda. Revista de Geografía Norte Grande, 35, 7-28. https://doi. org/10.4067/S0718-34022006000100002

Rowe, F. (2014). Micro and macro drivers of long-distance commuting in Chile: The role of spatial distribution of economic activities and population. Paper presented at the 53rd Western Regional Science Association, San Diego, CA, USA.

Schiappacasse, P., Contreras, M., y Fuensalida, C. (2001). Migraciones internas hacia la Región Metropolitana de Santiago de Chile: una comparación con planteamientos teóricos. Investigaciones Geográficas, 35, 1-25. https://doi. org/10.5354/0719-5370.2013.27735

Seidman, S. (1983). Network structure and minimum degree. Social Networks, 5(3), 269-287. https://doi. org/10.1016/0378-8733(83)90028-X

Shannon, P., Markiel, A., Baliga, N., Wang, J., Ramage, D., Amin, N., ... Ideker, T. (2003). Cytoscape: a software environment for integrated models of biomelecular interaction networks. Genome 
Res., 13(11), 2498-2504. https://doi.org/10.1101/ gr.1239303

Taylor, P.J. (2017). Promiscous agglomerations: towards Integrating urban agglomerations with urban networks. GaWC Research Bulletin, (457). Recuperado de http://www.lboro.ac.uk/gawc/rb/rb457.html

Zelinsky, W. (1971). The hypothesis of mobility transition. Geographical Review, 61(2), 219-249. https:// doi.org/10.2307/213996 